\title{
Association of sarcopenic obesity and body composition with metabolically unhealthy overweight/obese phenotypes among Iranian women: A cross-sectional study
}

\author{
Aliyu Tijani Jibril \\ Tehran University of Medical Sciences \\ Atieh Mirzababaei \\ Tehran University of Medical Sciences \\ Farideh Shiraseb \\ Tehran University of Medical Sciences \\ Niloufar Rasaei \\ Tehran University of Medical Sciences \\ Khadijeh - Mirzaei ( $\sim$ Mirzaei_kh@tums.ac.ir) \\ Tehran University of Medical Sciences https://orcid.org/0000-0003-3020-4285
}

\section{Research}

Keywords: Sarcopenic obesity, body composition, metabolic healthy obesity, metabolic unhealthy obesity

Posted Date: December 10th, 2020

DOI: https://doi.org/10.21203/rs.3.rs-122982/v1

License: (c) (1) This work is licensed under a Creative Commons Attribution 4.0 International License.

Read Full License 


\section{Abstract \\ Background}

Obesity is a major risk factor for metabolic syndrome, with its prevalence has increased over the past decade. Major changes in body composition with aging have a significant effect on many clinical outcomes. Sarcopenic obesity consists of both the presence of abnormal adipose tissue with a deficit of muscle mass.

\section{Methods}

This cross-sectional study was conducted among a total of 241 overweight/obese women aged between 18-48 years in Tehran, Iran, registered by the use of the multistage cluster random sampling method. Blood samples were taken following standard procedures. Body composition was assessed through a multi-frequency bioelectrical impedance analyzer. The metabolic risk was assessed according to Karelis criteria and the subjects were classified as either MHO or MUO phenotypes.

\section{Results}

Of the 241 subjects in this study (average age 35.32 years), 176 (73.03\%) were classified as MUO phenotype. Based on this study, the prevalence of sarcopenic obesity was $7.88 \%$. We found that high fatfree mass was more strongly and significantly associated with MUO phenotype. Furthermore, we found that individuals with high fat-free mass and high skeletal muscle mass had a significantly low prevalence of MUO phenotype. A significant positive correlation between metabolic phenotypes and sarcopenic obesity was also observed after all potential covariates were adjusted for.

\section{Conclusion}

These results of this study suggest that increased adiposity and decreased skeletal muscle mass are associated with unfavorable metabolic traits among overweight and obese Iranian women. SO was also found to be associated with a greater risk of developing MUO phenotype.

\section{Background}

Obesity has been defined according to the World Health Organisation (WHO) as an abnormal accumulation of fat that affects health negatively (1). Obesity is a major risk factor for metabolic and cardiovascular morbidity and mortality, and its prevalence has doubled since 1980 in middle-aged and older adults (2). Individuals who are obese are at greater risk of developing the metabolic disease compared to individuals of normal weight (3), while obesity has also been related to the incidence of various cancers, respiratory disorders, arteriosclerosis, arthritis, and fatty liver (4). According to a study by 
Fallahzadeh and associates on estimating the prevalence and trends of obesity in the Iranian population, it was reported that $16.5 \%$ of Iranian subjects older than 18 years were obese (5). Furthermore, as part of the Jahrom Health Study (JHS), $24.8 \%$ of women aged 30 years and above were obese (6). In obese women, it has been reported that a small decline in muscle strength may cause difficulties in bearing their excess weight and in moving efficiently (7). Obese subjects have been reported to have heterogeneous phenotypes with different degrees of metabolic risk (8). A sub-group of obese individuals without associated metabolic complications have been phenotypically described as metabolically healthy obese (MHO) individuals $(9,10)$. On the other hand, is the metabolically unhealthy obese individuals characterized by obesity-related metabolic complications (11). Previous studies have reported MHO phenotype to be associated with a healthy metabolic profile, characterized by a lower amount of visceral adipose tissue (VAT) and liver fat (12), favorable lipid profile, high insulin sensitivity, and low proinflammatory cytokine levels in plasma $(12,13)$ than metabolically unhealthy obese (MUO) subjects. Growing evidence however has shown that healthy obese adults without metabolic risk factors may progress to unhealthy obesity over time (14). Schroder et al., in a 10-year follow-up of Spanish obese cohort patients, found half of their subjects with an $\mathrm{MHO}$ phenotype at the beginning acquire an unhealthy phenotype (15). There is not a universal criterion to determine MUO and MHO, however, some studies have shown that BMI, blood pressure, high-density lipoprotein cholesterol, low-density lipoprotein cholesterol, triglyceride, fasting blood glucose, c-reactive protein, and homeostatic model assessment of insulin resistance (HOMA-IR) can be used as a criterion to diagnose obesity phenotype (3).

Aging is a continuous process characterized by a decline in most physiological functions (16). Major changes associated with aging in body composition include a decline in both fat-free mass and muscle strength, which has been referred to as sarcopenia (17); and, an increase in body fat and a decline in skeletal muscle, referred to as sarcopenic obesity (SO) (18). Sarcopenia and obesity are both related to metabolic disorders, morbidity, and mortality (19). It has therefore been hypothesized that sarcopenic obesity may have a greater impact on metabolic diseases and cardiovascular morbidity and mortality than either sarcopenia or obesity alone $(2,20)$. Sarcopenic obesity has been linked to increased production of inflammatory substances such as leptin, IL-6, tumor necrosis factor [TNF] a, IL-1a known to alter insulin sensitivity, growth hormone secretion, and energy metabolism $(18,21)$. Furthermore, Chung et al., in a study of elderly Koreans found that the sarcopenic obese group was more closely associated with insulin resistance, metabolic syndrome, and cardiovascular disease risk factors than any other group in both men and women (22). Moreover, numerous studies have also reported a significantly higher risk of mortality (23) worse cardiovascular risk profiles including hyperglycemia, hypertension, dyslipidemia, insulin resistance, and lower cardiorespiratory fitness $(22,24,25)$ in sarcopenic obese individuals compared to non-sarcopenic or non-obese subjects.

In a study of the Iranian adult population, Rahmanian and colleagues reported a $10.9 \%$ and a $7.2 \%$ prevalence of MUO and MHO respectively in this group (26). Rasaei and associates also reported a $9.9 \%$ prevalence of SO among Tehranian overweight and obese women (27). Despite the high prevalence of SO and $\mathrm{MUO}$ in this population, information regarding their association with body composition is scarce. The aim of this study therefore was to determine the association of metabolically healthy and unhealthy 
obesity with sarcopenic obesity and measures of body composition among overweight and obese Iranian women.

\section{Methods}

\section{Study sample}

This cross-sectional study was conducted among 241 overweight and obese women who were referred to health centers affiliated to Tehran University Medical Science in Iran. Subjects were registered by use of the multistage cluster random sampling method. All participants answered a questionnaire developed and administered by well-trained investigators Inclusion criteria were age 18-48 years, being overweight or obese (body mass index $(\mathrm{BMl}) \geq 25 \mathrm{~kg} / \mathrm{m}^{2}$, and not being pregnant. Exclusion criteria included having; an acute or chronic inflammatory disease, regular use of medication, history of hypertension, cardiovascular disease, diabetes mellitus, impaired renal and liver function, intake of alcohol or drug abuse, smoking, thyroid disease, malignancies, pregnancy, and lactation. Also, individuals with chronic diseases affecting their dietary intake as well as those following a special dietary pattern over the past year were excluded from this study and whose daily energy consumption was $800 \mathrm{kcal} /$ day or 4200 $\mathrm{kcal} /$ day were registered. All participants were informed about the study aims and procedures and provided written informed consent. The Medical Research Ethics Committee of Tehran University of Medical Sciences approved the study with the following identification IR.TUMS.VCR.REC.1398.692.

\section{Measurements}

\section{Dietary Assessment}

A semi-quantitative food frequency questionnaire (FFQ) which contains 147 -item food was applied to evaluate usual dietary intake and nutritional status over the past year. Household methods were used to transform portion sizes of the used up foods into grams (28), and a predictable average daily intake of food parameters (including macro and micronutrients) was computed from the FFQ using NUTRITIONIST IV software (version 7.0; N-Squared Computing, Salem, OR), modified for Iranian foods. This procedure was designed according to the Willett study including a list of 147 food items along with a standard serving size for each nutrient which its reliability and validity had been approved in Iran (29).

\section{Measurement of blood samples}

Blood samples were collected following overnight fasting and the serum was centrifuged, liquated, and stored at a temperature of $-80^{\circ} \mathrm{C}$ until the analysis was performed. All samples were analyzed using a single assay according to the manufacturer's protocol. The GOD PAP method and the GPO-PAP method were used for measuring the respective fasting blood sugar (FBS) and triglyceride (TG) amounts. Total cholesterol (T-chol) and cholesterol with high-density lipoprotein (HDL) were calculated using the enzymatic endpoint process and the enzymatic clearance assay. All calculations were made with the usage of a package from Randox Laboratories (Hitachi 902). An immunoturbidimetric assay (high 
sensitivity assay, Hitachi 902) was used to test high-sensitive C-reactive protein (hs-CRP). All measurements were taken at the Endocrinology and Metabolism Research Institute (EMRI) - Bionanotechnology Laboratory of Tehran University of Medical Science.

\section{Body composition and anthropometric measurements assessment}

Bioelectrical impedance analyzer (BIA) (InBody 720, South Korea) was used to calculate body composition indicators, including body fat-free mass and fat mass. This bioelectrical impedance analyzer (BIA) is considered to emit a very weak current to assess body impedance (resistance). Anthropometric measurements were performed on all participants, such as weight, BMI, waist circumference (WC), and waist-to-hip ratio (WHR). Weight was measured with digital scales and recorded to the nearest $0.1 \mathrm{~kg}$ while the subjects were in minimal clothing and without shoes. Height was measured using a tape measure while the subjects were standing erect, without shoes, and with shoulders in a normal position and, head, shoulders, heels, and buttocks are in contact with the wall. BMI was calculated by dividing the weight $(\mathrm{kg})$ by the square of the height $(\mathrm{m})$. Waist circumference $(\mathrm{WC})$ and hip circumference $(\mathrm{HC})$ were recorded to the nearest $0.1 \mathrm{~cm}$ in the central point of the iliac crest and rib cage with tape with an accuracy of $0.1 \mathrm{~cm}$. To reduce error, all measurements were taken by the same technician. All measurements were carried out by professionally trained technicians and according to specific guidelines to reduce the difference between subjects.

\section{Assessment of blood pressure}

The blood pressure was assessed after the participants rested for 10 minutes. Hypertension was defined as blood pressure $>130 / 85 \mathrm{mmHg}$. This category includes patients taking antihypertensive medicines, even if treatment achieves a blood pressure level that is within the target range.

\section{The HOMA-IR calculation}

The insulin resistance homeostatic model assessment (HOMA-IR) was based on the following equation: [fasting plasma glucose $(\mathrm{mmol} / \mathrm{l}) \times$ fasting plasma insulin $(\mathrm{mlU} / \mathrm{l})] / 22.5(30)$.

\section{Definition of metabolic health and sarcopenic obesity}

The Karelis criteria were used because all inflammation criteria are tested. The existence of four or more of the following five is described as metabolic abnormality according to the requirements of Karelis, composition: TG $1.7 \mathrm{mmol} / \mathrm{l}, \mathrm{HDL} 1.3 \mathrm{mmol} / \mathrm{l}$, without treatment; LDL $2.6 \mathrm{mmol} / \mathrm{l}$, without treatment, 3.0 $\mathrm{mg} / \mathrm{l} \mathrm{hs}-\mathrm{CRP}$ and 2.7 HOMA-IR (31). According to the definition, sarcopenic obesity in women is referred to as two lower quintiles of SMM and two highest quintiles of FM (32). In this study, we measured SMM and FM by BIA (Inbody Co., Seoul, Korea) according to guidelines.

\section{Other baseline measurements}


The socio-demographic and lifestyle characteristics assessed included age, gender, educational level attained, physical activity status, and smoking habits by questionnaire. A validated International Physical Activity Questionnaire (IPAQ) was used to assess the level of physical activity. For weekly physical activity assessment, a short form (9 items) was applied using MET (metabolic equivalent) scores for each type of activity. For reporting the total physical activity from all activity categories, MET scores across all these sub-components were summed and MET-minutes per week (MET-min/wk) were estimated (33).

\section{Statistical Analysis}

All statistical analyses were performed using the IBM SPSS software version 25.0 (SPSS, Chicago, IL, USA), and p-values less than 0.05 were considered statistically significant. Normal distribution of data was checked by the Kolmogorov-Smirnov test. Continuous variables information was represented by mean \pm standard deviations (SDs) and mean \pm standard errors (SEs), and categorical feature information represented by percentage and number. An independent sample t-test was used for assessed differences between groups with the Metabolically Healthy and Metabolically Healthy obese and chi-square $\left(\chi^{2}\right)$ tests for categorical variables. Analysis of covariance (ANCOVA) was used for adjusting potential confounders such as age, BMI, physical activity, and energy intake. Spearman bivariate correlation between body composition variables and sarcopenic obesity was performed. Association between metabolic healthy statuses with measures of body composition and sarcopenic obesity were assessed by the Binary logistic regression model, adjust for confounders in crude and 2 adjust models. Results were presented as beta and odds ratios (ORs) and $95 \%$ confidence intervals (Cls) compared with the metabolic unhealthy status group.

\section{Results}

\section{General characteristics of study participants}

The general characteristics of the study subjects according to metabolically healthy and unhealthy phenotypes were shown in Table1. In total, 241 women aged $18-48$ years, mean \pm SD age of $35.32 \pm 8.68$ years, participated in our study. Among these, $78.99 \%$ were married and $1.26 \%, 10.50 \%, 38.66 \%$, and $49.58 \%$, respectively, were illiterate, under diploma education level, diploma education level, and bachelor and a higher academic degree. A significant difference was observed among the study participants in terms of their economic status $(p=0.02)$. After controlling for potential confounders (age, physical activity (PA), energy intake, and BMI, this significance disappeared. The prevalence of sarcopenic obesity was $7.88 \%$. The aforementioned demographic characteristics and prevalence of sarcopenic obesity were marginally different across the two groups $(p=0.06)$. However, after adjusting for potential confounders, a significant difference was observed between the two groups $(p=0.04)$. Subjects in the MUO group had a significantly higher body weight, BMI, WC, body fat mass (BFM), and visceral fat area (VFA) compared to subjects in the metabolically healthy $(\mathrm{MHO})$ group $(p<0.001)$. Similar findings were observed after adjusting for potential confounding variables. The FFM, fat mass index (FMI), and skeletal muscle mass 
(SMM) were significantly higher in the MUO group, $p=0.004, p=0.001$, and $p=0.002$ respectively. FBS was seen to be significantly higher in the MUO group after potential confounders were controlled for $(p=0.03) . T G, L D L$, and total cholesterol were significantly higher $(p<0.001)$ in the unhealthy group, while the levels of $\mathrm{HDL}$ were significantly higher $(p<0.001)$ in the healthy group compared with the unhealthy group. After controlling for potential confounders, the results remained unchanged. A significant difference was also observed among the groups based on HOMA-IR $(p<0.001)$ and insulin level $(p=0.001)$, with the observed outcome remaining unchanged even after confounders were controlled for. While a non-significant difference was observed across the groups base on systolic blood pressure (SBP), a significantly higher diastolic blood pressure (DBP) was observed among the unhealthy group $(p<0.001)$. (hs-CRP levels in the MUO group was significantly higher than in the MHO group ( $p<0.001)$. We, however, did not observe a statistically significant difference in physical activity among the two groups $(p=0.33)$.

Table 1. General characteristics of study participants 


\section{variables}

Metabolically

Healthy

(mean $\pm S D)$

\section{Demographic characteristic}

Age (year) ${ }^{a}$

$35.92 \pm 7.55$

$17(34.0)$

47 (25.0)

0.21

0.80

Married (\%)

\section{Education ${ }^{b}$}

Illiterate (\%)

Under diploma (\%)

Diploma (\%)

Bachelor and above (\%)

\section{Economic status $^{b}$}

Low class (\%)

Middle class (\%)

High class (\%)

\section{Sarcopenic obesity ${ }^{b}$}

Non-sarcopenic obese (\%)

$61(27.5)$

20 (48.8)

$7(29.2)$

25 (80.6)

0.02

0.16

$21(51.2)$

$17(70.8)$

$1(33.3)$

$2(66.7)$

0.57

0.73

$21(84.0)$

$68(73.9)$

$83(70.3)$
P- P

value $^{*} \quad$ value $^{\ddagger^{\star \star}}$
$4(16.0)$

$24(26.1)$

$35(27.7)$

$6(19.4)$

)

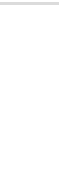

(a)

$33(66.0)$

Metabolically

$141(75.0)$
$161(72.5)$

0.06

0.04

\section{Sarcopenic obese (\%) \\ Anthropometry and Body Composition}

$4(21.1)$

\begin{tabular}{lcccc} 
Weight $(\mathrm{kg})^{\text {a }}$ & $74.89 \pm 8.71$ & $81.03 \pm 11.02$ & $<0.001$ & $<0.001$ \\
\hline Height $(\mathrm{cm})^{a}$ & $161.09 \pm 5.18$ & $161.51 \pm 5.83$ & 0.06 & 0.92 \\
\hline BMI $\left(\mathrm{kg} / \mathrm{m}^{2}\right)^{a}$ & $28.75 \pm 2.86$ & $31.11 \pm 3.64$ & $<0.001$ & $<0.001$ \\
WC $(\mathrm{cm})^{\text {a }}$ & $93.38 \pm 7.01$ & $99.64 \pm 9.27$ & $<0.001$ & $<0.001$ \\
\hline Body fat mass $(\mathrm{kg})^{\text {a }}$ & $29.76 \pm 5.96$ & $34.03 \pm 7.34$ & $<0.001$ & $<0.001$ \\
\hline Visceral fat area $\left(\mathrm{cm}^{2}\right)^{\text {a }}$ & $14.04 \pm 2.89$ & $15.72 \pm 3.25$ & $<0.001$ & 0.001
\end{tabular}




\begin{tabular}{|c|c|c|c|c|}
\hline Fat-free mass $(\mathrm{kg})^{\text {a }}$ & $44.84 \pm 4.68$ & $47.10 \pm 5.57$ & 0.004 & 0.01 \\
\hline Skeletal muscle mass $(\mathrm{kg})^{\mathrm{a}}$ & $24.42 \pm 2.69$ & $25.89 \pm 3.32$ & 0.002 & 0.008 \\
\hline Fat free mass index $(\mathrm{kg})^{a}$ & $17.26 \pm 1.21$ & $18.75 \pm 9.88$ & 0.23 & 0.27 \\
\hline Fat mass index $(\mathrm{kg})^{a}$ & $11.65 \pm 2.62$ & $13.06 \pm 2.78$ & 0.001 & $<0.001$ \\
\hline \multicolumn{5}{|l|}{ Blood Parameters } \\
\hline $\mathrm{FBG}(\mathrm{mg} / \mathrm{dL})^{a}$ & $4.68 \pm 0.40$ & $4.86 \pm 0.49$ & 0.05 & 0.03 \\
\hline $\mathrm{TG}(\mathrm{mg} / \mathrm{dL})^{\mathrm{a}}$ & $0.90 \pm 0.28$ & $1.52 \pm 0.82$ & $<0.001$ & $<0.001$ \\
\hline $\mathrm{HDL}(\mathrm{mg} / \mathrm{dL})^{\mathrm{a}}$ & $1.34 \pm 0.24$ & $1.14 \pm 0.26$ & $<0.001$ & $<0.001$ \\
\hline $\operatorname{LDL}(\mathrm{mg} / \mathrm{dL})^{\mathrm{a}}$ & $2.19 \pm 0.50$ & $2.53 \pm 0.62$ & $<0.001$ & 0.001 \\
\hline Total cholesterol $(\mathrm{mg} / \mathrm{dL})^{a}$ & $4.37 \pm 0.74$ & $4.89 \pm 0.93$ & $<0.001$ & 0.003 \\
\hline Insulin (mIU/l) & $1.13 \pm 0.24$ & $1.24 \pm 0.22$ & 0.001 & 0.01 \\
\hline HOMA index ${ }^{a}$ & $2.47 \pm 0.85$ & $3.66 \pm 1.24$ & $<0.001$ & $<0.001$ \\
\hline \multicolumn{5}{|l|}{ Blood Pressure } \\
\hline $\mathrm{SBP}(\mathrm{mmHg})^{a}$ & $109.01 \pm 12.44$ & $112.66 \pm 13.92$ & 0.06 & 0.18 \\
\hline $\mathrm{DBP}(\mathrm{mmHg})^{a}$ & $73.74 \pm 8.29$ & $79.39 \pm 10.05$ & $<0.001$ & 0.007 \\
\hline \multicolumn{5}{|c|}{ Inflammatory parameter and other variables } \\
\hline $\mathrm{Hs}-\mathrm{CRP}(\mathrm{mg} / \mathrm{L})^{a}$ & $1.33 \pm 1.18$ & $5.37 \pm 4.91$ & $<0.001$ & $<0.001$ \\
\hline $\begin{array}{l}\text { Physical activity (MET } \\
\text { min/week) }{ }^{a}\end{array}$ & $989.33 \pm 1078.53$ & $1304.14 \pm 2404.33$ & 0.33 & 0.29 \\
\hline
\end{tabular}

SD, standard deviation; BMI, body mass index; WC, waist circumference; FBG, fasting blood glucose; TG, triglyceride; HDL, high-density lipoprotein; LDL, low-density lipoprotein; hs-CRP, High sensitive c-reactive protein; SBP, systolic blood pressure; DBP, diastolic blood pressure.

Data are presented as mean \pm standard deviation (SD) or percent.

${ }^{\ddagger}$ Collinear variables did not enter into the model and this P-value obtained from ANCOVA analysis ${ }^{*}$-value obtained from independent T-test

** p-value obtained from ANCOVA test, variable adjust for age, physical activity, energy intake, and BMI 
Chi-square and analysis of variance were used for qualitative and quantitative variables respectively, and the $\mathrm{p}$-value was set to $<0.05$.

a Mean \pm SD

b Sample size (\%)

\section{Dietary intake of study subjects according to metabolic statuses}

As shown in Table 2, the food groups, mean dietary intakes, and the mean nutrient intakes of the study participants were not statistically significantly different between the $\mathrm{MHO}$ and $\mathrm{MUO}$ groups even after controlling for potential confounding variables $(p>0.05)$.

Table 2. Dietary intake of study subjects according to metabolic statuses 


\begin{tabular}{|c|c|c|c|c|c|c|}
\hline \multirow[t]{2}{*}{ Variables } & \multicolumn{2}{|c|}{ Metabolic healthy } & \multicolumn{2}{|c|}{ Metabolic unhealthy } & \multirow[t]{2}{*}{ P-value ${ }^{*}$} & \multirow[t]{2}{*}{ P-value ${ }^{* \star}$} \\
\hline & Mean & SD & Mean & SD & & \\
\hline \multicolumn{7}{|l|}{ Food groups } \\
\hline Fruits $(\mathrm{g} / \mathrm{d})$ & 508.45 & 373.30 & 505.83 & 352.66 & 0.96 & 0.34 \\
\hline Vegetables(g/d) & 379.99 & 227.11 & 387.94 & 248.29 & 0.82 & 0.12 \\
\hline Cereal(g/d) & 422.18 & 195.27 & 427.86 & 226.56 & 0.86 & 0.61 \\
\hline Whole grains(g/d) & 58.64 & 53.41 & 65.77 & 62.43 & 0.42 & 0.31 \\
\hline Refined grains(g/d) & 363.54 & 203.95 & 362.45 & 211.23 & 0.97 & 0.43 \\
\hline $\operatorname{Nuts}(g / d)$ & 15.23 & 16.91 & 19.06 & 28.03 & 0.96 & 0.67 \\
\hline Legumes(g/d) & 51.64 & 55.38 & 48.23 & 34.54 & 0.57 & 0.62 \\
\hline Red meat (g/d) & 21.61 & 17.70 & 22.53 & 19.75 & 0.74 & 0.60 \\
\hline White meat(g/d) & 38.34 & 32.02 & 47.34 & 42.68 & 0.12 & 0.54 \\
\hline Salt and salty snacks(g/d) & 36.79 & 38.78 & 41.18 & 47.54 & 0.51 & 0.99 \\
\hline Dairy(g/d) & 274.24 & 234.54 & 301.84 & 214.28 & 0.39 & 0.70 \\
\hline Tea(g/d) & 637.96 & 463.59 & 793.25 & 912.36 & 0.19 & 0.19 \\
\hline \multicolumn{7}{|l|}{ Dietary intake } \\
\hline Energy intake (kcal/d) & 2505.26 & 727.89 & 2615.62 & 754.66 & 0.31 & - \\
\hline Carbohydrate (g/d) & 362.65 & 126.42 & 370.55 & 117.74 & 0.65 & 0.20 \\
\hline Carbohydrate (\% energy) & 57.16 & 6.58 & 56.57 & 6.64 & 0.54 & 0.44 \\
\hline Protein $(\mathrm{g} / \mathrm{d})$ & 83.30 & 26.04 & 89.26 & 28.26 & 0.14 & 0.58 \\
\hline Protein (\% energy) & 13.43 & 2.61 & 13.75 & 2.33 & 0.36 & 0.64 \\
\hline Fat $(g / d)$ & 88.66 & 27.49 & 94.91 & 33.61 & 0.18 & 0.33 \\
\hline Fat (\% energy) & 32.38 & 5.79 & 32.75 & 6.66 & 0.82 & 0.62 \\
\hline MUFA (g/d) & 28.93 & 8.47 & 31.39 & 11.69 & 0.12 & 0.24 \\
\hline PUFA(g/d) & 18.84 & 6.71 & 20.12 & 9.06 & 0.30 & 0.64 \\
\hline $\operatorname{SFA}(g / d)$ & 25.99 & 9.76 & 28.43 & 11.60 & 0.13 & 0.14 \\
\hline Fiber (g/d) & 45.18 & 20.05 & 44.30 & 17.86 & 0.74 & 0.15 \\
\hline \multicolumn{7}{|l|}{ Nutrients } \\
\hline Vitamin A (RAE) & 729.58 & 351.47 & 795.26 & 428.82 & 0.27 & 0.84 \\
\hline
\end{tabular}




\begin{tabular}{|lllllll|}
\hline Thiamine $(\mathrm{mg} / \mathrm{d})$ & 2.01 & 0.65 & 2.05 & 0.63 & 0.63 & 0.39 \\
\hline Riboflavin $(\mathrm{mg} / \mathrm{d})$ & 2.05 & 0.71 & 2.23 & 0.84 & 0.14 & 0.30 \\
\hline Niacin $(\mathrm{mg} / \mathrm{d})$ & 23.59 & 7.09 & 25.25 & 9.30 & 0.19 & 0.74 \\
\hline Vitamin B6 $(\mathrm{mg} / \mathrm{d})$ & 2.06 & 0.67 & 2.17 & 0.72 & 0.28 & 0.80 \\
\hline Folic acid $(\mu \mathrm{g} / \mathrm{d})$ & 585.20 & 181.26 & 606.54 & 171.92 & 0.40 & 0.66 \\
\hline Vitamin B12 $(\mu \mathrm{g} / \mathrm{d})$ & 3.99 & 2.00 & 4.47 & 2.36 & 0.15 & 0.29 \\
\hline Vitamin $\mathrm{C}(\mathrm{mg} / \mathrm{d})$ & 190.26 & 124.40 & 195.71 & 132.75 & 0.77 & 0.19 \\
\hline Vitamin $\mathrm{E}(\mathrm{mg} / \mathrm{d})$ & 15.91 & 6.73 & 17.32 & 9.28 & 0.26 & 0.37 \\
\hline Vitamin D $(\mu \mathrm{g} / \mathrm{d})$ & 2.05 & 1.73 & 1.87 & 1.47 & 0.44 & 0.22 \\
\hline Iron $(\mathrm{mg} / \mathrm{d})$ & 18.28 & 6.03 & 18.63 & 5.90 & 0.68 & 0.14 \\
\hline Selenium $(\mu \mathrm{g} / \mathrm{d})$ & 114.81 & 36.86 & 119.67 & 42.80 & 0.42 & 0.95 \\
\hline Zinc $(\mathrm{mg} / \mathrm{d})$ & 12.33 & 4.13 & 13.12 & 4.30 & 0.20 & 0.94 \\
\hline Magnesium $(\mathrm{mg} / \mathrm{d})$ & 443.71 & 144.98 & 463.73 & 150.39 & 0.36 & 0.65 \\
\hline Calcium $(\mathrm{mg} / \mathrm{d})$ & 1117.99 & 423.03 & 1158.55 & 410.52 & 0.50 & 0.82 \\
\hline Potassium $(\mathrm{mg} / \mathrm{d})$ & 4208.43 & 1662.90 & 4345.83 & 1538.23 & 0.55 & 0.44 \\
\hline Caffeine $(\mathrm{g} / \mathrm{d})$ & 147.13 & 105.55 & 157.53 & 178.52 & 0.66 & 0.72 \\
\hline & & & & & & \\
\hline
\end{tabular}

MUFA, monounsaturated fatty acid; PUFA, polyunsaturated fatty acid; SFA, Saturated fatty acid Data are presented as mean \pm standard deviation (SD).

${ }^{a}$ Calculated by analysis of variance and $p$-value $<0.05$ indicates a significant level.

*Obtained from Independent T-test

**Obtained from ANCOVA analysis

Food group adjusted by age, BMI, physical activity, and energy intake (Kcal)

Dietary intake (macronutrient and micronutrient) adjusted by energy intake (Kcal)

\section{Correlation of main variables with sarcopenic obesity}

Table 3 contains the correlation between sarcopenic obesity with the variables of interest in this study. A simple correlation revealed a significant inverse associations between FFM and FFMI with correlation coefficient $(r=-0.30, p=<0.001)$ and $(r=-0.12, p=0.01)$ respectively with sarcopenic obesity, while significant 
positive correlations were observed between BFM $(r=0.27, p=<0.001)$ and FMI $(r=0.33, p=<0.001)$ with sarcopenic obesity. A non-significant association was however observed between metabolic healthy obesity statuses with sarcopenic obesity.

Table 3. Spearman bivariate correlation of main variables with sarcopenic obesity

\begin{tabular}{|lll|}
\hline Variables & Spearman's correlation coefficient $(r)$ & P-value \\
\hline Fat Free Mass & $-0.30^{\star \star}$ & $<0.001$ \\
\hline Body Fat Mass & $0.27^{\star \star}$ & $<0.001$ \\
\hline FFMI & $-0.12^{\star}$ & 0.01 \\
\hline FMI & $0.33^{\star \star}$ & $<0.001$ \\
\hline Metabolic Healthy Obesity & 0.04 & 0.45 \\
\hline
\end{tabular}

Spearman Correlation Analysis

FFMI, fat-free mass index; FMI, fat mass index

**. Correlation is significant at the 0.01 level (2-tailed).

*. Correlation is significant at the 0.05 level (2-tailed).

\section{Association between obesity statuses with measures of body composition}

With the binary logistic regression analysis, beta $(\beta)$, odds ratio $(\mathrm{OR})$, and confidence interval $(\mathrm{Cl})$ for the association of sarcopenic obesity with measures of body composition in crude and adjusted models are presented in Table 4. In the crude model, participants with MUO had greater odds of increasing FFM ( $\beta=$ $0.76, \mathrm{OR}=0.27,95 \% \mathrm{Cl}=0.13-0.56, p=0.001)$ than the $\mathrm{MHO}$ subjects. However, after controlling for age, total energy intake, and physical activity level, the MUO groups had 0.29 folds decreased odds of FFM ( $\beta=-0.89, \mathrm{OR}=0.29,95 \% \mathrm{Cl}=0.13-0.62, p=0.008$ ) compared to the $\mathrm{MHO}$ group. After additionally controlling for weight and economic status, the observed association disappeared $(\beta=-0.55, \mathrm{OR}=0.26,95 \% \mathrm{Cl}=0.11$ $0.59, p=0.07)$. In the crude model, participants with MUO were observed to be positively associated with greater odds of increasing BFM ( $\beta=1.31$, OR: $3.73,95 \% \mathrm{Cl}=1.27-10.91, p=0.01)$ than those with MHO. Also, after controlling for age, total energy intake, and physical activity level, subjects with MUO phenotype had 4.44 greater odds for $B F M$, compared to those with $\mathrm{MHO}$ phenotype $(\beta=1.49, \mathrm{OR}=4.44,95 \% \mathrm{Cl}=1.31$ $15.00, p=0.01)$. Even after additional control for weight and economic status, the positive association of the MUO score with higher odds for the BFM remained significant $(\beta=0.95, O R=2.60,95 \% \mathrm{Cl}=1.39-17.20$, $p=0.02$ ). Subjects with MUO phenotype in the crude model were observed to have a $0.87 \mathrm{~kg}$ lower SMM compared with the $\mathrm{MHO}$ subjects $(\beta=-0.87, \mathrm{OR}=0.50,95 \% \mathrm{Cl}=0.27-0.90, p=0.006)$. After age, total energy intake, and physical activity had been controlled for, the MUO participants had a 0.83 lower SMM compared with the $\mathrm{MHO}$ subjects $(\beta=-0.83, \mathrm{OR}=0.46,95 \% \mathrm{Cl}=0.22-0.84, p=0.01)$. Furthermore, after 
additional adjustment for weight and economic status, the association remained significant $(\beta=-0.73$, $\mathrm{OR}=0.47,95 \% \mathrm{Cl}=0.24-0.94, p=0.03)$. In the crude model, a non-significant association was seen between metabolic healthy statuses with sarcopenic obesity scores $(\beta=0.35, \mathrm{OR}=1.42,95 \% \mathrm{Cl}=0.45-4.45, p=0.54)$. However, after controlling for age, total energy intake, physical activity level, weight, and economic status, we observed MUO subjects to have 4.21 fold greater odds for sarcopenic obesity compared to the MHO subjects $(\beta=1.43, \mathrm{OR}=4.21,95 \% \mathrm{Cl}=1.28-62.26, p=0.02)$.

Table 4. Association between metabolic healthy statuses with measures of body composition and sarcopenic obesity 


\begin{tabular}{|c|c|c|c|c|}
\hline Variables & & $\beta$ & OR $(95 \% \mathrm{Cl})$ & P-value \\
\hline \multicolumn{5}{|c|}{ Fat-Free Mass } \\
\hline \multirow[t]{2}{*}{ Crude } & MUO & 0.76 & $0.27(0.13,0.56)$ & 0.001 \\
\hline & $\mathrm{MHO}$ & Ref & Ref & \\
\hline \multirow[t]{2}{*}{ Model 1} & MUO & -0.89 & $0.29(0.13,0.62)$ & 0.008 \\
\hline & $\mathrm{MHO}$ & Ref & Ref & \\
\hline \multirow[t]{2}{*}{ Model 2} & MUO & -0.55 & $0.26(0.11,0.59)$ & 0.07 \\
\hline & $\mathrm{MHO}$ & Ref & Ref & \\
\hline \multicolumn{5}{|c|}{ Body Fat Mass } \\
\hline \multirow[t]{2}{*}{ Crude } & MUO & 1.31 & $3.73(1.27,10.91)$ & 0.01 \\
\hline & $\mathrm{MHO}$ & Ref & Ref & \\
\hline \multirow[t]{2}{*}{ Model 1} & MUO & 1.49 & $4.44(1.31,15.00)$ & 0.01 \\
\hline & $\mathrm{MHO}$ & Ref & Ref & \\
\hline \multirow[t]{2}{*}{ Model 2} & MUO & 0.95 & $2.60(1.39,17.20)$ & 0.02 \\
\hline & $\mathrm{MHO}$ & Ref & Ref & \\
\hline \multicolumn{5}{|c|}{ Skeletal Muscle Mass } \\
\hline \multirow[t]{2}{*}{ Crude } & MUO & -0.87 & $0.50(0.27,0.90)$ & 0.006 \\
\hline & $\mathrm{MHO}$ & Ref & Ref & \\
\hline \multirow[t]{2}{*}{ Model 1} & MUO & -0.83 & $0.46(0.22,0.84)$ & 0.01 \\
\hline & $\mathrm{MHO}$ & Ref & Ref & \\
\hline \multirow[t]{2}{*}{ Model 2} & MUO & -0.73 & $0.47(0.24,0.94)$ & 0.03 \\
\hline & $\mathrm{MHO}$ & Ref & Ref & \\
\hline \multicolumn{5}{|c|}{ Sarcopenic obesity } \\
\hline \multirow[t]{2}{*}{ Crude } & MUO & 0.35 & $1.42(0.45,4.45)$ & 0.54 \\
\hline & $\mathrm{MHO}$ & Ref & Ref & \\
\hline \multirow[t]{2}{*}{ Model 1} & MUO & 0.42 & $1.52(0.40,5.70)$ & 0.53 \\
\hline & $\mathrm{MHO}$ & Ref & Ref & \\
\hline \multirow[t]{2}{*}{ Model 2} & MUO & 1.43 & $4.21(1.28,62.26)$ & 0.02 \\
\hline & $\mathrm{MHO}$ & Ref & Ref & \\
\hline
\end{tabular}


MUO; metabolic unhealthy obese, $\mathrm{MH}$; metabolic obese

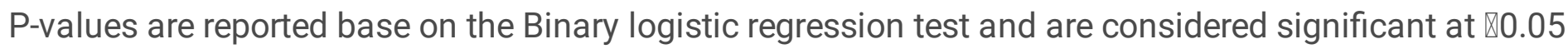

Healthy obese is a reference group

Model 1: Adjusted for age, kcal, and physical activity

Model 2: Model 1 confounders with further adjustment with weight and economic status

\section{Discussion}

This study was conducted on overweight and obese Iranian women who were between the ages of 18-50 years, with a BMI greater than or equal to $25 \mathrm{~kg} / \mathrm{m}^{2}$. The metabolic risk was assessed according to Karelis criteria and the subjects were classified as either MHO or MUO. Of the 241 subjects in this study (average age 35.32 years), 176 (73.03\%) were classified as MUO phenotype. Based on this study, the prevalence of sarcopenic obesity was $7.88 \%$. Our study found that high fat-free mass was more strongly and significantly associated with MUO phenotype. Furthermore, we found that individuals with high fatfree mass and high skeletal muscle mass had a significantly low prevalence of MUO phenotype. Consistently, the significant association between high SMM and MUO phenotype persisted even after all potential confounders (age, total energy intake, physical activity, weight, and economic status) were controlled for. Also, we found a significant positive correlation between metabolic phenotypes with sarcopenic obesity after all potential covariates were adjusted for.

Previous epidemiological studies have addressed the issue of fat mass, muscle mass, and sarcopenic obesity in relation to metabolic phenotypes in both Asian and Western populations. The body composition analysis of the two phenotype groups ( $\mathrm{MUO}$ and $\mathrm{MHO}$ ) showed that BFM, VFM, FFM, SMM, and $\mathrm{FMI}$, as measured by $\mathrm{BIA}$, were significantly higher in the MUO group than in the MHO group. Previous studies have found that MUO women had significantly higher body fat mass, visceral fat mass, and muscle mass than MHO women [21] and healthy obese people [29].

Despite the fact that some studies $(9,34,35)$ have observed a non-significant difference in the measures of adiposity in "healthy" and "unhealthy" phenotypes, in line with other previous studies $(3,10)$, we observed a significant increase in body fat mass in the MUO phenotype even after all potential confounders were controlled for. Body fat mass has been reported as an independent predictor of insulin resistance and dyslipidemia (36) among postmenopausal women. Moreover, higher body fat has been reported as an increased risk factor for type 2 diabetes $(37,38)$, as well as increase metabolic risk and the risk of cardiovascular disease $(39,40)$ in both men and women. Furthermore, researches have shown increasing body fat to be correlated with an increase in fasting blood glucose, triglyceride, LDL cholesterol, and total cholesterol, and a decrease in $\mathrm{HDL}$ cholesterol $(41,42)$. 
There have been conflicting results regarding SMM accumulation among the metabolic phenotype groups. While in some studies, no significant differences in SMM indices between metabolic phenotypes of either obese or non-obese postmenopausal women (35) were found, some studies have reported SMM to significantly increase in the MUO phenotype of postmenopausal women $(11,43)$, and significantly decrease in the metabolic non-obese phenotype of young women (5). Also, Estrella et al., (44) among Hispanic/Latino women, found higher lean body mass to be independently associated with a lower prevalence of the MHO phenotype. In our study, however, each increase of 1-SD in skeletal muscle mass was associated with a lower prevalence of the MUO phenotype. The result from the fourth and fifth Korean National Health and Nutrition Examination Survey indicated a protective association of muscle mass with metabolic syndrome, which was attenuated by high-fat mass (45). Additionally, Burrows et al. found in a Chilean birth cohort that low muscle mass is associated with cardiometabolic risk factors independent of dietary intake (46). Furthermore, in a 7-year retrospective cohort study, Kim and colleagues (47) found that an increase in relative skeletal muscle mass over time has a potential preventive effect on developing metabolic syndrome, independently of baseline skeletal muscle mass and glycometabolic parameters. In a nationally representative sample of 4,449 US adults aged 50 years and older from the NHANES surveys, Li and associates (48) found that only participants with low muscle mass but without metabolic syndrome had a significantly increased risk of all-cause mortality. Earlier studies have reported maintenance of muscle mass in the elderly as crucial, as it serves as a metabolic reservoir that is needed to effectively withstand disease $(49,50)$. The outcome of our study supports the suggestion from a previous report about the importance of skeletal muscle mass in glucose regulation (51), and thus its protection from the development of cardiometabolic abnormalities (45).

After all potential confounders were adjusted for, each increase of 1-SD in sarcopenic obesity was found to be associated with a higher prevalence of MUO phenotype in the current study. Earlier epidemiological studies have shown that the odds of metabolic syndrome was 6 to 8 times higher in postmenopausal Korean women, elderly Korean men and women, and adult Caucasian subjects with sarcopenic obesity (SO) compared to those without sarcopenic obesity (24, 52-54). Furthermore, Kim et al., (55) reported an increased risk of metabolic syndrome in subjects with sarcopenic obesity. In their study, compared with normal subjects among women, they found that SO subjects had significantly higher values for a number of metabolic syndrome components. Furthermore, they found $\mathrm{SO}$ to be independently associated with metabolic syndrome among women after adjustment for age.

\section{Strengths And Limitations}

Our study has inherent strengths and limitations. A validated food frequency questionnaire was used to assess participants' usual dietary intake. Also, all measurements and interviews were conducted by trained personnel.

Even though this study provides valuable information regarding metabolic phenotypes with sarcopenic obesity and measures of body composition among Iranian subjects, we acknowledge limitations that need to be addressed. First, the cross-sectional nature of this study does not permit the assessment of 
causality. Only a prospective study would provide a better understanding of the observed associations. Another limitation of our study is that we used BIA in order to assess body composition and not dual Xray absorptiometry (DXA) which is considered the gold standard method. However, BIA is also a validated and reliable method for the measurement of body composition in the adult population (56). Furthermore, the result of the current study cannot be generalized since only females were included in the study.

\section{Conclusion}

In conclusion, increased adiposity and decreased skeletal muscle mass was associated with unfavorable metabolic traits among overweight and obese Iranian women. SO was also found to be associated with a greater risk of developing MUO phenotype after controlling for potential confounders.

\section{Abbreviations}

BFM body fat mass

BIA Bioelectrical impedance analyzer

BMI body mass index

Cl confidence intervals

DBP diastolic blood pressure

DXA dual X-ray absorptiometry

FBS fasting blood sugar

FFM fat-free mass

FFMl fat-free mass index

FFQ food frequency questionnaire

FM fat mass

FMl fat mass index

HC hip circumference

HDL high-density lipoprotein

HOMA-IR homeostatic model assessment of insulin resistance hs-CRP high-sensitive C-reactive protein 
IPAQ International Physical Activity Questionnaire

JHS Jahrom Health Study

LDL low-density lipoprotein

MET metabolic equivalent

MHO metabolically healthy obese

MUO metabolically unhealthy obese

OR odds ratios

SBP systolic blood pressure

SDs standard deviations

SE standard errors

SMM skeletal muscle mass

SO sarcopenic obesity

T-chol Total cholesterol

TG triglyceride

TNF tumor necrosis factor

VAT visceral adipose tissue

WC waist circumference

WHO World Health Organization

WHR waist-to-hip ratio

\section{Declarations}

\section{Ethical approval}

This study was approved by the ethics committee of Tehran University of Medical Sciences (TUMS) with the following identification IR.TUMS.VCR.REC.1398.692. 
Not applicable.

\section{Availability of data and materials}

Participants of this study did not agree for their data to be shared publicly, so supporting data is not available.

\section{Competing interests}

All authors declared that they have no competing interests

\section{Funding}

This study is funded by grants from the Tehran University of Medical Sciences (TUMS). (Grant ID: 97-03161-41155).

\section{Author's contributions}

ATJ wrote the article, AM and NR revised the article, FSH performed the statistical analyses, KhM had full access to all of the data in the study and took responsibility for the integrity and accuracy of the data. All authors read and approved the final manuscript.

\section{Acknowledgments}

The authors thank the study participants for their cooperation and assistance in physical examinations. This study was supported by TUMS.

\section{References}

1. Ser RoaWcJWHOTR. Obesity: preventing and managing the global epidemic. 2000;894(i-xii):1-253.

2. Wannamethee SG, Atkins JLJPotNS. Muscle loss and obesity: the health implications of sarcopenia and sarcopenic obesity. 2015;74(4):405-12.

3. Kang EY, Yim J-EJNR, Practice. Differences in dietary intakes, body compositions, and biochemical indices between metabolically healthy and metabolically abnormal obese Korean women. 2019;13(6):488-97.

4. Kweon S, Kim Y, Jang M-j, Kim Y, Kim K, Choi S, et al. Data resource profile: the Korea national health and nutrition examination survey (KNHANES). 2014;43(1):69-77.

5. Fallahzadeh H, Saadati H, Keyghobadi NJSJ. Estimating the prevalence and trends of obesity in Iran populations from 2000 to 2011: a meta-analysis study. 2017;25(9):681-9.

6. Shojaei M, Jahromi AS, Rahmanian K, Madani AJOJoBS. Gender differences in the prevalence of cardiovascular risk factors in an Iranian urban population. 2015;15(3):178-84. 
7. Stenholm S, Harris TB, Rantanen T, Visser M, Kritchevsky SB, Ferrucci LJCoicn, et al. Sarcopenic obesity-definition, etiology and consequences. 2008;11(6):693.

8. Vecchié A, Dallegri F, Carbone F, Bonaventura A, Liberale L, Portincasa P, et al. Obesity phenotypes and their paradoxical association with cardiovascular diseases. 2018;48:6-17.

9. Brochu M, Tchernof A, Dionne IJ, Sites CK, Eltabbakh GH, Sims EA, et al. What are the physical characteristics associated with a normal metabolic profile despite a high level of obesity in postmenopausal women? 2001;86(3):1020-5.

10. Karelis AD, Faraj M, Bastard J-P, St-Pierre DH, Brochu M, Prud'homme D, et al. The metabolically healthy but obese individual presents a favorable inflammation profile. 2005;90(7):4145-50.

11. Wildman RP, Muntner P, Reynolds K, McGinn AP, Rajpathak S, Wylie-Rosett J, et al. The obese without cardiometabolic risk factor clustering and the normal weight with cardiometabolic risk factor clustering: prevalence and correlates of 2 phenotypes among the US population (NHANES 19992004). 2008;168(15):1617-24.

12. Primeau V, Coderre L, Karelis A, Brochu M, Lavoie M, Messier V, et al. Characterizing the profile of obese patients who are metabolically healthy. 2011;35(7):971-81.

13. Naukkarinen J, Heinonen S, Hakkarainen A, Lundbom J, Vuolteenaho K, Saarinen L, et al. Characterising metabolically healthy obesity in weight-discordant monozygotic twins. 2014;57(1):167-76.

14. Gaita D, Mosteoru SJCE, Metabolism. Metabolically healthy versus unhealthy obesity and risk for diabetes mellitus and cardiovascular diseases. 2017;6(1):23-6.

15. Schröder H, Ramos R, Baena-Díez JM, Mendez MA, Canal DJ, Fíto M, et al. Determinants of the transition from a cardiometabolic normal to abnormal overweight/obese phenotype in a Spanish population. 2014;53(6):1345-53.

16. Batsis JA, Mackenzie TA, Lopez-Jimenez F, Bartels SJJNr. Sarcopenia, sarcopenic obesity, and functional impairments in older adults: National Health and Nutrition Examination Surveys 19992004. 2015;35(12):1031-9.

17. Rosenberg IJAJCN. Epidemiologic and methodologic problems in determining nutritional status of older persons.(Summary comments). 1989;50:1231-3.

18. Baumgartner RNJAotNYAoS. Body composition in healthy aging. 2000;904(1):437-48.

19. Zamboni M, Mazzali G, Fantin F, Rossi A, Di Francesco VJN, Metabolism, Diseases C. Sarcopenic obesity: a new category of obesity in the elderly. 2008;18(5):388-95.

20. Kohara KJE. Sarcopenic obesity in aging population: current status and future directions for research. 2014;45(1):15-25.

21. Baumgartner RN, Wayne SJ, Waters DL, Janssen I, Gallagher D, Morley JEJOr. Sarcopenic obesity predicts instrumental activities of daily living disability in the elderly. 2004;12(12):1995-2004.

22. Chung J-Y, Kang H-T, Lee D-C, Lee H-R, Lee Y-JJAog, geriatrics. Body composition and its association with cardiometabolic risk factors in the elderly: a focus on sarcopenic obesity. 2013;56(1):270-8. 
23. Wannamethee SG, Shaper AG, Lennon L, Whincup PHJTAjocn. Decreased muscle mass and increased central adiposity are independently related to mortality in older men. 2007;86(5):1339-46.

24. Lim S, Kim JH, Yoon JW, Kang SM, Choi SH, Park YJ, et al. Sarcopenic obesity: prevalence and association with metabolic syndrome in the Korean Longitudinal Study on Health and Aging (KLoSHA). 2010;33(7):1652-4.

25. Kim TN, Park MS, Kim YJ, Lee EJ, Kim M-K, Kim JM, et al. Association of low muscle mass and combined low muscle mass and visceral obesity with low cardiorespiratory fitness. 2014;9(6):e100118.

26. Rahmanian K, Shojaei M, Jahromi ASJD, metabolic syndrome, targets o, therapy. Prevalence and clinical characteristics of metabolically unhealthy obesity in an Iranian adult population. 2019;12:1387.

27. Rasaei N, Kashavarz SA, Yekaninejad MS, Mirzaei KJD, Research MSC, Reviews. The association between sarcopenic obesity (SO) and major dietary patterns in overweight and obese adult women. 2019;13(4):2519-24.

28. Ghaffarpour M, Houshiar-Rad A, Kianfar HJTNOK. The manual for household measures, cooking yields factors and edible portion of foods. 1999;7:213.

29. Mirmiran P, Hosseini-Esfahani F, Jessri M, Mahan LK, Shiva N, Azizi F. Does dietary intake by Tehranian adults align with the 2005 dietary guidelines for Americans? Observations from the Tehran lipid and glucose study. Journal of health, population, and nutrition. 2011;29(1):39.

30. Matthews D, Hosker J, Rudenski A, Naylor B, Treacher DJD. Turner RC. Homeostasis model assessment: insulin resistance and beta-cell function from fasting plasma glucose and insulin concentrations in man. 1985;28(7):412-9.

31. Hinnouho G-M, Czernichow S, Dugravot A, Batty GD, Kivimaki M, Singh-Manoux AJDc. Metabolically healthy obesity and risk of mortality: does the definition of metabolic health matter? 2013;36(8):2294-300.

32. Chen L-K, Liu L-K, Woo J, Assantachai P, Auyeung T-W, Bahyah KS, et al. Sarcopenia in Asia: consensus report of the Asian Working Group for Sarcopenia. 2014;15(2):95-101.

33. Craig CL, Marshall AL, Sjöström M, Bauman AE, Booth ML, Ainsworth BE, et al. International physical activity questionnaire: 12 -country reliability and validity. Medicine \& science in sports \& exercise. 2003;35(8):1381-95.

34. Messier V, Karelis AD, Robillard M-Ė, Bellefeuille P, Brochu M, Lavoie J-M, et al. Metabolically healthy but obese individuals: relationship with hepatic enzymes. 2010;59(1):20-4.

35. Peppa M, Koliaki C, Papaefstathiou A, Garoflos E, Katsilambros N, Raptis SA, et al. Body composition determinants of metabolic phenotypes of obesity in nonobese and obese postmenopausal women. 2013;21(9):1807-14.

36. Van Pelt R, Evans E, Schechtman K, Ehsani A, Kohrt WJAJoP-E, Metabolism. Contributions of total and regional fat mass to risk for cardiovascular disease in older women. 2002. 
37. Snijder MB, Dekker JM, Visser M, Bouter LM, Stehouwer CD, Yudkin JS, et al. Trunk fat and leg fat have independent and opposite associations with fasting and postload glucose levels: the Hoorn study. 2004;27(2):372-7.

38. Choi SI, Chung D, Lim JS, Lee MY, Shin JY, Chung CH, et al. Relationship between regional body fat distribution and diabetes mellitus: 2008 to 2010 Korean National Health and Nutrition Examination Surveys. 2017;41(1):51-9.

39. Okura T, Nakata Y, Yamabuki K, Tanaka KJA, Thrombosis,, Biology V. Regional body composition changes exhibit opposing effects on coronary heart disease risk factors. 2004;24(5):923-9.

40. Madeira FB, Silva AA, Veloso HF, Goldani MZ, Kac G, Cardoso VC, et al. Normal weight obesity is associated with metabolic syndrome and insulin resistance in young adults from a middle-income country. 2013;8(3):e60673.

41. Bi X, Seabolt L, Shibao C, Buchowski M, Kang H, Keil C, et al. DXA-measured visceral adipose tissue predicts impaired glucose tolerance and metabolic syndrome in obese Caucasian and AfricanAmerican women. 2015;69(3):329-36.

42. Aucouturier J, Meyer M, Thivel D, Taillardat M, Duché PJAop, medicine a. Effect of android to gynoid fat ratio on insulin resistance in obese youth. 2009;163(9):826-31.

43. Davies MJ, Baer DJ, Judd JT, Brown ED, Campbell WS, Taylor PRJJ. Effects of moderate alcohol intake on fasting insulin and glucose concentrations and insulin sensitivity in postmenopausal women: a randomized controlled trial. 2002;287(19):2559-62.

44. Estrella ML, Pirzada A, Durazo-Arvizu RA, Cai J, Giachello AL, Espinoza Gacinto R, et al. Correlates of and Body Composition Measures Associated with metabolically healthy obesity phenotype in hispanic/latino women and men: the Hispanic Community Health Study/Study of Latinos (HCHS/SOL). 2019;2019.

45. Kim K, Park SMJSr. Association of muscle mass and fat mass with insulin resistance and the prevalence of metabolic syndrome in Korean adults: a cross-sectional study. 2018;8(1):1-8.

46. Burrows R, Correa-Burrows P, Reyes M, Blanco E, Albala C, Gahagan SJPd. Low muscle mass is associated with cardiometabolic risk regardless of nutritional status in adolescents: A crosssectional study in a Chilean birth cohort. 2017;18(8):895-902.

47. Kim G, Lee S-E, Jun JE, Lee Y-B, Ahn J, Bae JC, et al. Increase in relative skeletal muscle mass over time and its inverse association with metabolic syndrome development: a 7-year retrospective cohort study. 2018;17(1):1-13.

48. Li R, Xia J, Zhang X, Gathirua-Mwangi WG, Guo J, Li Y, et al. Associations of muscle mass and strength with all-cause mortality among US older adults. 2018;50(3):458.

49. Srikanthan P, Horwich TB, Tseng CHJTAjoc. Relation of muscle mass and fat mass to cardiovascular disease mortality. 2016;117(8):1355-60.

50. Atkins JL, Whincup PH, Morris RW, Lennon LT, Papacosta O, Wannamethee SGJJotAGS. Sarcopenic obesity and risk of cardiovascular disease and mortality: a population-based cohort study of older men. 2014;62(2):253-60. 
51. Brooks N, Layne JE, Gordon PL, Roubenoff R, Nelson ME, Castaneda-Sceppa CJljoms. Strength training improves muscle quality and insulin sensitivity in Hispanic older adults with type 2 diabetes. 2007;4(1):19.

52. Kim TN, Park MS, Lim Kl, Yang SJ, Yoo HJ, Kang HJ, et al. Skeletal muscle mass to visceral fat area ratio is associated with metabolic syndrome and arterial stiffness: the Korean Sarcopenic Obesity Study (KSOS). 2011;93(2):285-91.

53. Kang S-Y, Lim GE, Kim YK, Kim HW, Lee K, Park T-J, et al. Association between sarcopenic obesity and metabolic syndrome in postmenopausal women: a cross-sectional study based on the Korean National Health and Nutritional Examination Surveys from 2008 to 2011. 2017;24(1):9-14.

54. Poggiogalle E, Lubrano C, Sergi G, Coin A, Gnessi L, Mariani S, et al. Sarcopenic obesity and metabolic syndrome in adult Caucasian subjects. 2016;20(9):958-63.

55. Kim TN, Yang S, Yoo H-J, Lim K, Kang H, Song W, et al. Prevalence of sarcopenia and sarcopenic obesity in Korean adults: the Korean sarcopenic obesity study. 2009;33(8):885-92.

56. Kim H, Kim C-H, Kim D-W, Park M, Park HS, Min S-S, et al. External cross-validation of bioelectrical impedance analysis for the assessment of body composition in Korean adults. 2011;5(3):246-52. 